\title{
Thrush in infancy
}

Candida albicans can be found in many adults as a saprophyte in the gastrointestinal tract and probably other sites, but apart from vaginal candidiasis it is unusual for a frank infection to occur unless the adult host is compromised by a severe illness, by failure of the immunological mechanism, or during treatment with cytotoxic drugs. In newborns and infants, however, the story is different and cases of clinical thrush occur frequently. The actual incidence is difficult to assess because authors do not always indicate their diagnostic criteria so that in the literature incidences varying from $0.5 \%$ to nearly $20 \%$ have been recorded for various nurseries. Not all white patches in the mouth are due to infection with $C$. albicans though the organisms might be grown from a swab. Rogers ${ }^{1}$ has suggested that thrush should only be diagnosed if a scraping of the epithelium shows the formation of hyphae. This procedure is seldom done except perhaps in the case of skin infections.

The reasons for this very wide variation in incidence may be differences in practice between hospitals rather than differences in diagnostic criteria. For instance, it has been clearly shown by several authors, and in particular Shrand, ${ }^{2}$ that if a mother has vulvo-vaginitis due to $C$. albicans which is incompletely treated or not treated at all the risk of thrush in the infant is quite high. He found that when the mothers did not have candida vaginitis less than $1 \%$ of their babies developed thrush, whereas with inadequate or no treatment of the vaginitis, between 60 and $70 \%$ of the babies developed oral thrush. It is therefore important when assessing the significance of a reported incidence of thrush in newborn infants to know what kind of antenatal treatment the mothers are given. Most cases of oral thrush in newborn infants occur in intensive care units, though it has been shown that there is no direct link with prematurity. ${ }^{2}$ Nearly all such infants have been in the nursery for more than 7 days and it is uncommon to detect clinical thrush within a few days of birth. ${ }^{2}$ If a hospital discharges a large proportion of mothers and babies within 48 hours the incidence of thrush will be very low. There is also the variation in the practice of individual paediatricians: if the babies are inspected carefully every day and swabs are taken of any suspicious patches the incidence of thrush will probably be higher than in the nursery where babies are not examined so thoroughly.

As well as infecting the mouth, $C$. albicans can cause a dermatitis of the napkin area and many of the infants with candida skin lesions who excrete C. albicans in their stool also have positive mouth swabs, though not necessarily all show clinical oral thrush. Infections with C. albicans in the well newborn tend to be of a self-limiting nature even without treatment, but with active treatment usually clear in a few days. However, in the sick neonate severe candida infection not only interferes with normal feeding but also in a few cases progresses to systemic disease, so that the physician in charge of any group of infants at risk must be prepared to act at the first signs of candida infection 'and the problem is one of deciding that a true infection of the tissues exists.

Russell and Lay ${ }^{3}$ studied 140 term normal infants and followed the colonization of their mouths by candida species. The isolation rate of candida rose from $5.7 \%$ on the first day of life to $14.2 \%$ on discharge from hospital at 7 days, and increased sharply after the infants had returned home, reaching $82 \%$ at 4 weeks, thereafter declining slowly to $50 \%$ at 1 year. In this series there were 28 babies colonized with $C$. albicans itself at 4 weeks of life and only 3 developed clinical thrush. In a further series reported in this issue, Lay and Russell ${ }^{4}$ studied 99 babies who were in a special care unit. The rate of isolation of candida was similar to the more normal babies in that $6 \%$ were colonized in the first day of life, rising to $53 \%$ on day 14 . After discharge from hospital colonization rose to $79 \%$ at 4 weeks and fell to $50 \%$ at 1 year of age. 17 of these babies had C. albicans at 4 weeks and 4 developed clinical thrush. This is a somewhat higher incidence than in the normal infants, $24 \%$ as opposed to $10 \%$, but the numbers are very small. A small group of 29 babies who had been in incubators were also studied but the isolation rates were very similar. Although the initial and final colonization rates were similar, the actual rate of colonization was in fact much more rapid in infants in the special care unit than in the normal infants previously studied so that on day $7,30 \%$ of the infants in the special care unit were colonized as against $14 \%$ of the normal infants. This suggests that 
in a situation like the neonatal intensive care unit cross infection with candida plays a part, and suggests that there is a problem of hygiene. ${ }^{5,6}$

Isolated cases of oral thrush in newborn infants are probably nearly always the result of infection from the vagina of the mother, though probably very few babies of mothers who carry candida develop the disease. Dawkins ${ }^{7}$ examined 201 mouth cultures of babies whose mothers had been shown to harbour C. albicans during pregnancy and only one swab was positive, and this mother had been found repeatedly to have positive swabs. In St. Mary's Hospital, Manchester, in 1976 there were 323 mothers known to have $C$. albicans in the vagina during pregnancy, but only 3 of the babies of these mothers were noted to have oral thrush while in hospital. When there is active disease in the mother the incidence is higher, ${ }^{2}$ and Bound ${ }^{8}$ also found a significantly increased incidence of thrush vaginitis in mothers of babies who developed a candida napkin rash. $27 \%$ of mothers of babies with napkin rash had thrush vaginitis. About half the infants in this series developed the rashes at home and less than one-third showed oral thrush. This suggested that the infection was being transferred to the infant's skin by the mother's hands.

If a large number of cases occur in a nursery it is probably an indication of cross infection. In one reported series 530 out of 52 babies in two preterm nurseries developed oral thrush in a period of a few weeks. Only $20 \%$ of the mothers were positive and it was clearly shown that the majority of these babies with oral thrush excreted candida in stools and 14 developed skin rashes due to $C$. albicans. There was also heavy contamination of the environment. Conditions such as these in sick baby nurseries can lead to more serious infection.

Infections have occurred with candida on the surface of neural tube defects and even at the sites of venous or arterial cannula. There is the added complication that this could give rise to contamination of laboratory specimens causing a wrong diagnosis of meningitis or septicaemia due to C. albicans, though true cases of these serious systemic infections can occur. Many systemic infections are only diagnosed at necropsy, and Kozinn and Taschdjian ${ }^{9}$ drew attention to the frequency of enteric candidiasis with lesions in the small intestines including invasion of the mucous membrane by mycelia. Rogers ${ }^{1}$ also described cases of enteritis apparently due to $C$. albicans and noted that the infection may be primary in sick children or may supervene after initial infection by other intestinal pathogens. It can be present without evidence of oral thrush and at necropsy invasion of the gut is seen. Clinically the diarrhoea responds well to oral nystatin. Bishop et al. ${ }^{10}$ found
C. albicans in the stomach and duodenum of onethird of the infants suffering from gastroenteritis, and growth in the duodenum was associated with depression of lactase activity which may have been either primary or secondary. Recently Bishop and Barnes $^{11}$ have shown that the $C$. albicans primarily depresses lactase activity in the small gut in the infant rabbit, though there was no evidence of mycelial invasion by the yeast so that there must be damage to the intestinal epithelial cells by the growth of the organism in the gut lumen. This depression of lactase activity has interesting implications for the treatment of enteritis in infancy and one of its sequelae, disaccharide intolerance.

Attempts have been made to use other means of deciding whether an individual with the clinical signs of thrush is suffering from a true infection; these usually involve a search for antibody production. Russell and Lay ${ }^{3}$ looked for antibodies in the cord blood of the infants in their first series and found no correlation with initial or subsequent colonization. Stanley and Hurley ${ }^{12}$ studied precipitins to candida in pregnant women and found that $64 \%$ of the women in whom a firm diagnosis of C. albicans mycosis was made showed the presence of precipitins. None of the few newborn infants with oral thrush showed precipitins. Although these methods are useful in studying the natural history of the disease they are of little value in the situation which confronts the clinician with an individual case. It may be better to rely on the observations of many workers that the presence of candida in swabs is often followed later by frank candida infections.

Although it is obvious that in well babies $C$. albicans occurs commonly as a commensal and need not cause concern, the situation is not the same with ill babies or those in intensive care units where the early colonization of babies' mouths not only exposes them to a risk of clinical thrush infections and possibly systemic infections, but also may indicate that all is not well with the control of infection in the nursery. Of the 52 cases of clinical infections with C. albicans in St. Mary's Hospital in 1976 only 8 were in well babies; the remaining 44 occurred in the intensive care baby unit both in medical and surgical cases. Although the majority of these infections were of the mouth or skin, infections of urine, lung, and surgical wounds occurred. The risk of developing systemic, lung, or gut infections is also high in this special group of infants, so that even if it is often difficult to decide whether a true infection is present in an infant the risk to other babies in the nursery must always be remembered. The treatment of suspected thrush is so simple and free from risk that even in doubtful cases it seems reasonable to start treatment. 


\section{References}

${ }^{1}$ Rogers, K. B. (1966). Candida infections in paediatrics. Symposium on Candida Infections, p. 178. Ed. by H. I. Winner and R. Hurley. Livingstone, Edinburgh and London.

${ }^{2}$ Shrand, H. (1961). Thrush in the newborn. British Medical Journal, 2, 1530-1533.

${ }^{3}$ Russell, C., and Lay, K. M. (1973). Natural history of candida species and yeasts in the oral cavity of infants. Archives of Oral Biology, 18, 957-962.

'Lay, K. M., and Russell, C. (1977). Candida species and yeasts in the mouths of infants from a special care unit of a maternity hospital. Archives of Disease in Childhood, 52, 794-796.

Jennison, R. F. (1966). Candida infection in a maternity hospital. Symposium on Candida Infections, p. 102-112. Ed. by H. I. Winner and R. Hurley. Livingstone, Edinburgh and London.

'Blaschke-Hellmessen, R., Hinkel, G. K., and Kintzel, H. W. (1973). Zum Problem des Candida-Hospitalismus bei Frühgeborenen. Dermatologische Monatsschrift, 159, 403-409.

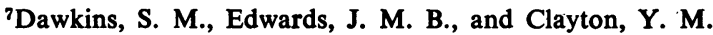
(1958). Candida albicans in vaginal secretions in pregnancy. Fungous Diseases and their Treatment, pp. 94-101. Ed. by R. W. Riddell and G. T. Stewart. Butterworth, London.

${ }^{8}$ Bound, J. P. (1958). Skin moniliasis in infants. Fungous Diseases and their Treatment, p. 102. Ed. by R. W. Riddell and G. T. Stewart. Butterworth, London.

${ }^{9}$ Kozinn, P. J., and Taschdjian, C. L. (1962). Enteric candidiasis. Diagnosis and clinical considerations. Pediatrics, 30, 71-85.

${ }^{10}$ Bishop, R. F., Barnes, G. L., and Townley, R. R. W. (1973). Microbial flora of stomach and small intestine in infantile gastroenteritis. Unpublished observations.

${ }^{11}$ Bishop, R. F., and Barnes, G. L. (1974). Depression of lactase activity in the small intestine of infant rabbits by C. albicans. Journal of Medical Microbiology, 7, 259-263.

${ }^{12}$ Stanley, V. C., and Hurley, R. (1974). Candida.precipitins in pregnant women: validity of the test systems used. Journal of Clinical Pathology, 27, 66-69.

R. F. JenNison

Department of Pathology, St. Mary's Hospital, Whitworth Park, Manchester M13 OJH. 